\title{
UNIVERSAL IMMERSION SPACES FOR EDGE-COLORED GRAPHS AND NEAREST-NEIGHBOR METRICS*
}

\author{
YAIR BARTAL ${ }^{\dagger}$ AND LEONARD J. SCHULMAN ${ }^{\ddagger}$
}

\begin{abstract}
There exist finite universal immersion spaces for the following: (a) Edge-colored graphs of bounded degree and boundedly many colors. (b) Nearest-neighbor metrics of bounded degree and boundedly many edge lengths.
\end{abstract}

Key words. universal graph, universal space, edge coloring, finite metric, homomorphism, immersion, embedding

AMS subject classifications. 05C12, 05C15, 51F15

DOI. $10.1137 / 08071555 \mathrm{X}$

1. Edge-colored graphs. An edge-colored graph $G=((V, E), \chi)$ is a graph with vertices $V$ and edges $E$ (undirected; no multiple edges or loops), whose edges are colored by the mapping $\chi$. (The coloring need not be proper in any sense.) If $G^{\prime}=\left(\left(V^{\prime}, E^{\prime}\right), \chi^{\prime}\right)$ is another edge-colored graph, an immersion $\eta: G \rightarrow G^{\prime}$ is a mapping $V \rightarrow V^{\prime}$ satisfying the following. (a) For every $v \in V, \eta$ restricts to an isomorphism from the induced subgraph on $v$ and its neighbors, to the induced subgraph on the image of that set of vertices (equivalently, the mapping exactly preserves distances of 1 and 2); (b) $\chi^{\prime} \eta=\chi$.

Let $\mathcal{G}_{k, C}$ be the set of edge-colored graphs in which the degree of every vertex is bounded by $k$ and edges are colored by the finite set $C$ of cardinality $c$.

THEOREM 1.1. There exists a finite edge-colored graph $U_{k, C}$ such that for every $G \in \mathcal{G}_{k, C}$ there is an immersion $\eta: G \rightarrow U_{k, C}$.

We refer to $U_{k, C}$ as a universal immersion graph (or space) for $\mathcal{G}_{k, C}$.

Proof. Let $T$ be the set of "pointed" edge-colored graphs (graphs with a distinguished vertex called the root) in which every vertex is within distance 1 of the root, the vertex degrees are bounded by $k$, and the edges are colored by the set $C$.

Introduce an auxiliary set of colors $\Xi$ of cardinality $k^{3}+k^{2}+k+1$. Let $\mathcal{V}$ be the set of all vertex colorings of edge-colored graphs in $T$ by $\Xi$, in which no vertex color is used twice. That is, $\mathcal{V}=\left\{(t, \chi, \xi):(t, \chi) \in T, \xi \in \Xi^{T}, \xi\right.$ is injective $\}$.

Fix $U_{k, C}=((\mathcal{V}, \mathcal{E}), \zeta)$, with edges $\mathcal{E}$ defined and colored by $\zeta$ as follows. Let $u_{1}, u_{2} \in \mathcal{V}$, with $u_{i}=\left(t_{i}, \chi_{i}, \xi_{i}\right)$. Then set $\left\{u_{1}, u_{2}\right\}$ to be in $\mathcal{E}$ if (a) $\operatorname{root}\left(t_{1}\right)$ is connected by an edge to a vertex of the same color as $\operatorname{root}\left(t_{2}\right),(\mathrm{b}) \operatorname{root}\left(t_{2}\right)$ is connected by an edge to a vertex of the same color as $\operatorname{root}\left(t_{1}\right)$, and (c) the subgraphs of $u_{1}$ and $u_{2}$, induced by the vertices whose colors occur in both graphs, are isomorphic (as edge- and vertex-colored graphs). Such an edge $\left\{u_{1}, u_{2}\right\}$ in $\mathcal{E}$ is assigned the edge color $\zeta\left(\left\{u_{1}, u_{2}\right\}\right)$ common to each of the edges described in items (a) and (b).

Let $G=((V, E), \chi) \in \mathcal{G}_{k, C}$. To immerse $G$ in $U_{k, C}$, begin by coloring $V$ using the colors $\Xi$ so that vertices at distance $\leq 3$ receive distinct colors. The desired immersion

*Received by the editors February 12, 2008; accepted for publication (in revised form) March 15, 2009; published electronically June 26, 2009. This work was supported in part by a grant from the Israeli Science Foundation (195/02), and in part by NSF CCF-0515342 and NSA H98230-06-1-0074. http://www.siam.org/journals/sidma/23-2/71555.html

†School of Engineering and Computer Science, Hebrew University, Israel and Center for the Mathematics of Information, Caltech, MC256-80, Pasadena, CA 91125 (yair@cs.huji.ac.il).

${ }^{\ddagger}$ Caltech, MC256-80, Pasadena, CA 91125 (schulman@caltech.edu). 
$\eta$ carries $v \in V$ to the unique $\left(t_{v}, \chi_{v}, \xi_{v}\right)$ in $\mathcal{V}$ for which the radius-1 neighborhood about $v$ is isomorphic as a pointed graph to $t_{v}$ (in particular, the isomorphism carries $v$ to the root of $t_{v}$ ), and in which the vertex and edge colorings agree under the isomorphism.

It remains to be seen what $\eta$ does to pairs of vertices $u, v \in G$ that are at distance 1 or 2 . To begin, the vertex coloring ensures that in either case, $\eta(u) \neq \eta(v)$. Next, if $u$ and $v$ are neighbors, we must verify that the same is true of $\eta(u)$ and $\eta(v)$. The vertex coloring ensures that no color occurs twice in the union of the neighborhoods of radius 1 about $u$ and $v$. A vertex color can therefore occur in both of the pointed graphs $\eta(u)$ and $\eta(v)$ only if it is the color of a vertex $w \in G$ that is connected to both $u$ and $v$. In this case both of the pointed graphs contain a triangle among the images of $u, v$, and $w$, and in addition, the edges of each of these triangles are colored as they are in $G$. Moreover, both pointed graphs must also agree on the existence and color of edges between the images of distinct vertices $w, w^{\prime}$ that are neighbors of both $u$ and $v$.

Finally, we must ensure that if $u$ and $v$ are at distance 2, then so are $\eta(u)$ and $\eta(v)$. The radius- 1 neighborhood of $u$ cannot contain any vertex with $v$ 's color. Hence, there is no vertex in the pointed graph $\eta(u)$ that shares the color of the root of the pointed graph $\eta(v)$. Consequently, there is no edge in $\mathcal{E}$ between $\eta(u)$ and $\eta(v)$.

Remarks. Embeddings: A much stronger notion than immersion is that of an embedding, which, in addition to preserving some local structure around every vertex, is required also to be injective on the vertices. There is extensive literature, initiated by Rado [10] (see also Rotman [11]), on universal or, more demandingly, induceduniversal graphs for specific families of graphs $\mathcal{F}$; these are graphs in which every member of $\mathcal{F}$ embeds as, respectively, a subgraph or an induced subgraph. Since these developments concern embeddings rather than immersions, we do not list them exhaustively, but see Butler [4] for a useful survey, and for a construction of the smallest known induced-universal graph for bounded-degree graphs on $n$ vertices.

Naturally one cannot ask for a finite universal or induced-universal graph for embeddings of an infinite family $\mathcal{F}$. Our result shows (even apart from the edgecoloring requirement) that the situation is very different for immersions.

The definition of immersion: The term (in keeping with its origins in differential geometry [12]) should imply that local structure is preserved, which begs the question of how much local structure. One could choose a weaker definition than ours, so that only distances of 1 need be preserved (distances of 2 might decrease): this is a graph homomorphism. Note that this allows neighbors of a vertex to be identified if the edges leading to them have the same color. It is slightly easier to obtain a universal immersion graph for edge-colored graphs with this definition, and Alon and Marshall [2] have done just this but prove more: they provide a universal immersion graph, in this weaker sense, for the set of edge-colored graphs whose edges are colored by $c$ colors and which have acyclic chromatic number (see [6]) bounded by $K$. Their universal graph has cardinality bounded by $K c^{K-1}$, which is almost tight. For large $k$ it is known that the greatest possible acyclic chromatic number of graphs of maximum degree $k$ is between $\Omega\left(k^{4 / 3} / \log ^{1 / 3} k\right)$ and $O\left(k^{4 / 3}\right)$ (see [3], and see Fertin and Raspaud [5] for a recent algorithm), so Alon and Marshall's result implies that for the weaker notion of immersion, degree- $k c$-edge-colored graphs have a universal immersion graph of size $k^{4 / 3} c^{O\left(k^{4 / 3}\right)}$. (In contrast to the abundance of work on universal graphs for embeddings, this is the only prior work we are aware of that provides a universal graph for immersions of a class of edge-colored graphs.) 
The size of our construction: $U_{k, C}$ has $(c+1)^{O\left(k^{2}\right)} k^{O(k)}$ vertices. (Count $(c+$ 1) ${ }^{O\left(k^{2}\right)}$ underlying radius-1 edge-colored graphs and $k^{O(k)}$ vertex colorings.) Principally, then, the cost of the stronger immersion (rather than homomorphism) requirement has been an exponent proportional to $k^{2}$ rather than $k^{4 / 3}$. However, the best known lower bound is yet smaller (Alon and Marshall's result is nearly tight as a function of acyclic chromatic number but not necessarily as a function of maximum degree); an argument of Moon [9] (given for the uncolored case but easily translated to the colored case) shows that $U_{k, C}$ must have at least $(c+1)^{(k-1) / 2}$ vertices.

2. Nearest-neighbor metrics. We now show the existence of a universal immersion space for a class of discrete metric spaces. Say that $G=\left((V, E), \chi^{*}\right)$ is a graph metric if:

(1) $(V, E)$ is a graph (with undirected edges and without multiple edges or loops), and $\chi^{*}$ is a positive real-valued function on unordered pairs of distinct vertices.

(2) Letting $\chi$ be the restriction of $\chi^{*}$ to $E, \chi^{*}$ is the shortest-path metric induced on $G$ by $\chi$, and $\chi(\{u, v\})=\chi^{*}(\{u, v\})$ for every edge $\{u, v\}$. (Triangle inequality.)

Let $G=\left((V, E), \chi^{*}\right)$ and $G=\left(\left(V^{\prime}, E^{\prime}\right), \chi^{\prime *}\right)$ be two graph metrics. An immersion $\eta: G \rightarrow G^{\prime}$ is a mapping $V \rightarrow V^{\prime}$ that carries $E$ into $E^{\prime}$ and such that $\chi^{\prime} \eta=\chi$. A universal immersion space for a class of graph metrics is one into which any of them can be immersed.

Say that a graph metric $G$ is a $k$-nearest-neighbor metric if:

(3) No $u \in V$ has more than $k$ neighbors.

(4) If $\{u, v\} \in E$, then either $u$ or $v$ has edges to all strictly closer vertices.

Conditions (3) and (4) can be satisfied in useful cases, e.g., smooth finite-dimensional manifolds that have been discretized by fairly uniform sampling. On the other hand, the conditions exclude, for example, metrics induced by star graphs of unbounded degree.

In what follows we also require, solely for technical reasons, that $|\operatorname{Im} \chi| \leq c$ for some finite parameter $c$. (That is, there are only a limited number of distinct nearestneighbor edge lengths.) This restriction is not always natural, but we discuss below its impact and ways around it.

Let $C$ be a set of $c$ positive reals, and let $\mathcal{M}_{k, C}$ be the set of $k$-nearest-neighbor metrics in which all edge lengths lie in $C$.

In this paper the word "distance" refers always to the number of edges on a path; such a path may traverse directed edges (which we introduce shortly) in either direction. The word "length" refers always to the numbers labeling the edges of a path, denoted always by $\chi$ or $\zeta$.

THEOREM 2.1. There exists a finite graph metric $M_{k, C}$ such that for every $G \in$ $\mathcal{M}_{k, C}$ there is an immersion $\eta: G \rightarrow M_{k, C}$.

Proof. The construction of $M_{k, C}$ is similar to but slightly more complicated than the construction of $U_{k, C}$ in the previous section. (It also suffices for the requirements of that section but is larger.) Let $T$ be the set of "pointed" edge-colored directed graphs $(t, \chi)$ (without multiple edges, loops, or cycles of two edges) such that every vertex is within distance 2 (irrespective of the direction of the edges) of root $(t)$, the vertex degrees are bounded by $k$ (again irrespective of the direction of the edges), the edges are colored by the set $C$, the induced shortest-path metric $\chi^{*}$ satisfies conditions (1)-(4) (again irrespective of the direction of the edges), and finally, if $(u, v)$ is a directed edge and $\chi^{*}(\{u, w\})<\chi^{*}(\{u, v\})$, then $\{u, w\}$ is an edge (in some 
direction). Observe that the direction of the edges plays a role only in the last clause, which forces condition (4) to be achieved on a specific side.

Introduce an auxiliary set of colors $\Xi$ of cardinality $k^{4}+k^{3}+k^{2}+k+1$. Let $\mathcal{V}$ be the set of all vertex colorings of edge-colored graphs in $T$ by $\Xi$, in which no vertex color is used twice. That is, $\mathcal{V}=\left\{(t, \chi, \xi):(t, \chi) \in T, \xi \in \Xi^{T}, \xi\right.$ is injective $\}$.

Given a directed edge- and vertex-colored graph $G=((V, E), \chi, \xi)$, and a set of vertices $W \subseteq V$, let $\left.G\right|_{W}$ denote the induced directed edge- and vertex-colored subgraph on $W$. For $r \geq 0$ and a vertex $v \in V$, let $N_{r}(v)$ denote the set of vertices within distance $r$ of $v$ (irrespective of the direction of edges).

Fix $M_{k, C}=((\mathcal{V}, \mathcal{E}), \zeta)$, with edges $\mathcal{E}$ defined and colored by $\zeta$ as follows. Let $u_{1}, u_{2} \in \mathcal{V}$, with $u_{i}=\left(t_{i}, \chi_{i}, \xi_{i}\right)$. Then set $\left\{u_{1}, u_{2}\right\}$ to be in $\mathcal{E}$ if there exists a neighbor $v_{12}$ of $\operatorname{root}\left(t_{1}\right)$ and a neighbor $v_{21}$ of $\operatorname{root}\left(t_{2}\right)$, such that the directed edgeand vertex-colored graphs $\left.t_{1}\right|_{N_{2}\left(v_{12}\right)}$ and $\left.t_{2}\right|_{N_{2}\left(v_{21}\right)}$ are isomorphic under some mapping carrying $\operatorname{root}\left(t_{1}\right)$ to $v_{21}$ and $v_{12}$ to $\operatorname{root}\left(t_{2}\right)$. Under these conditions the edge $\left\{u_{1}, u_{2}\right\}$ is assigned the color $\zeta\left(\left\{u_{1}, u_{2}\right\}\right)=\chi_{1}\left(\left\{\operatorname{root}\left(t_{1}\right), v_{12}\right\}\right)=\chi_{2}\left(\left\{\operatorname{root}\left(t_{2}\right), v_{21}\right\}\right)$. (Note that the edges of $M_{k, C}$ are necessarily colored but not directed.)

Finally, induce from $\zeta$ the shortest-path metric $\zeta^{*}$ on $M_{k, C}$.

We begin by showing that $M_{k, C}$ is a universal immersion space for edge-colored graphs (i.e., in the sense of the previous section). Let $G=((V, E), \chi) \in \mathcal{M}_{k, C}$. To immerse $G$ in $M_{k, C}$, begin by coloring $V$ using the colors $\Xi$ so that vertices at distance $\leq 4$ receive distinct colors; and by directing the edges of $G$ to satisfy condition (4). The desired immersion $\eta$ carries $v \in V$ to the unique $\left(t_{v}, \chi_{v}, \xi_{v}\right)$ in $\mathcal{V}$ for which the radius-2 neighborhood about $v$ is isomorphic as a pointed graph to $t_{v}$ (in particular the isomorphism carries $v$ to the root of $t_{v}$ ), and in which the vertex colorings, edge colorings, and edge directions agree under the isomorphism.

It remains to be seen what $\eta$ does to pairs of vertices $u, v \in G$ that are at distance 1 or 2 . To begin, the vertex coloring ensures that in either case, $\eta(u) \neq \eta(v)$. Next, if $u$ and $v$ are neighbors, we must verify that the same is true of $\eta(u)$ and $\eta(v)$. This holds because both $\eta(u)$ and $\eta(v)$, which are pointed graphs of radius 2 about their roots, contain induced copies of $\left.G\right|_{N_{2}(u) \cap N_{2}(v)}$. Finally, if $u$ and $v$ are at distance 2, $\eta(u)$ and $\eta(v)$ are not connected because the vertex-coloring of $G$ ensures that $u$ has no neighbor colored identically to $v$, hence $\operatorname{root}(\eta(u))$ has no neighbor with that color, which is also the color of $\operatorname{root}(\eta(v))$. (This argument requires that $G$ be colored only in order to prevent repetitions within distance 3.)

Next we turn to showing that $M_{k, C}$ is a universal immersion space for the set of graph metrics $\mathcal{M}_{k, C}$. Since we have already ensured that $\eta$ respects edge coloring (i.e., $\zeta \eta=\chi$ ), and since $\zeta^{*}$ is automatically a metric, what remains is to show that $\zeta=\zeta^{*}$ on the edges of $M_{k, C}$.

Supposing the contrary, let $\left(u_{1}, u_{2}, \ldots, u_{r}\right)$ be a minimal counterexample in the following sense: (a) $\left\{u_{1}, u_{r}\right\} \in \mathcal{E}$; (b) $\forall i\left\{u_{i}, u_{i+1}\right\} \in \mathcal{E}$; (c) $\sum_{i=1}^{r-1} \zeta\left(\left\{u_{i}, u_{i+1}\right\}\right)<$ $\zeta\left(\left\{u_{1}, u_{r}\right\}\right)$; and (d) $r$ is minimal with properties (a)-(c). Denote $u_{i}=\left(t_{i}, \chi_{i}, \xi_{i}\right)$.

Let $v_{1 r}$ be the vertex of $t_{1}$, and let $v_{r 1}$ be the vertex of $t_{r}$, such that the edges $\left\{\operatorname{root}\left(t_{1}\right), v_{1 r}\right\}$ and $\left\{\operatorname{root}\left(t_{r}\right), v_{r 1}\right\}$ are carried into each other by the isomorphism that establishes the edge $\left\{u_{1}, u_{r}\right\}$. (Naturally $\operatorname{root}\left(t_{1}\right)$ is carried to $v_{r 1}$ and $v_{1 r}$ is carried to root $\left(t_{r}\right)$.) Without loss of generality suppose that the direction of the edge $\left\{\operatorname{root}\left(t_{1}\right), v_{1 r}\right\}$ is $\left(\operatorname{root}\left(t_{1}\right), v_{1 r}\right)$. (Hence the direction of the edge $\left\{\operatorname{root}\left(t_{r}\right), v_{r 1}\right\}$ is $\left(v_{r 1}, \operatorname{root}\left(t_{r}\right)\right)$.)

Let $s$ be the largest index such that for all $2 \leq s^{\prime} \leq s, t_{s^{\prime}}$ contains two vertices $v_{s^{\prime} 1}, v_{s^{\prime} r}$ such that $v_{s^{\prime} 1}$ is a neighbor of $\operatorname{root}\left(t_{s^{\prime}}\right), \xi_{s^{\prime}}\left(v_{s^{\prime} 1}\right)=\xi_{1}\left(\operatorname{root}\left(t_{1}\right)\right), \xi_{s^{\prime}}\left(v_{s^{\prime} r}\right)=$ 
$\xi_{1}\left(v_{1 r}\right)$, and $\chi_{s^{\prime}}\left(\left\{v_{s^{\prime} 1}, v_{s^{\prime} r}\right\}\right)=\chi_{1}\left(\left\{\operatorname{root}\left(t_{1}\right), v_{1 r}\right\}\right)=\zeta\left(\left\{u_{1}, u_{r}\right\}\right)$. These conditions are satisfied for $s^{\prime}=2$, so $s \geq 2$.

Now we show that for all $s^{\prime} \leq s, \sum_{i=1}^{s^{\prime}-1} \zeta\left(\left\{u_{i}, u_{i+1}\right\}\right) \geq \chi_{s^{\prime}}\left(\left\{\operatorname{root}\left(t_{s^{\prime}}\right), v_{s^{\prime} 1}\right\}\right)$. For $s^{\prime}=2$ this follows (with equality) by definition. For $s^{\prime}>2$ let $v_{s^{\prime}, s^{\prime}-1}$ be the vertex within $t_{s^{\prime}}$ that is carried to $\operatorname{root}\left(t_{s^{\prime}-1}\right)$ by the isomorphism establishing the edge $\left\{u_{s^{\prime}-1}, u_{s^{\prime}}\right\}$. This isomorphism establishes also that $\chi_{s^{\prime}}\left(\left\{v_{s^{\prime}, s^{\prime}-1}, v_{s^{\prime}, 1}\right\}\right)=$ $\chi_{s^{\prime}-1}\left(\left\{\operatorname{root}\left(t_{s^{\prime}-1}\right), v_{s^{\prime}-1,1}\right\}\right)$; by induction this quantity is $\leq \sum_{i=1}^{s^{\prime}-2} \zeta\left(\left\{u_{i}, u_{i+1}\right\}\right)$. Finally, note that $\zeta\left(\left\{u_{s^{\prime}-1}, u_{s^{\prime}}\right\}\right)=\chi_{s^{\prime}}\left(\left\{\operatorname{root}\left(t_{s^{\prime}}\right), v_{s^{\prime}, s^{\prime}-1}\right\}\right)$, and that by the triangle inequality within $t_{s^{\prime}}, \chi_{s^{\prime}}\left(\left\{\operatorname{root}\left(t_{s^{\prime}}\right), v_{s^{\prime}, s^{\prime}-1}\right\}\right) \geq \chi_{s^{\prime}}\left(\left\{\operatorname{root}\left(t_{s^{\prime}}\right), v_{s^{\prime}, 1}\right\}\right)-\chi_{s^{\prime}}\left(\left\{v_{s^{\prime}, s^{\prime}-1}\right.\right.$, $\left.\left.v_{s^{\prime}, 1}\right\}\right)$.

Now there are two cases. If $s=r$, then $\sum_{i=1}^{r-1} \zeta\left(\left\{u_{i}, u_{i+1}\right\}\right) \geq \chi_{r}\left(\left\{\operatorname{root}\left(t_{r}\right), v_{r 1}\right\}\right)=$ $\zeta\left(\left\{u_{r}, u_{1}\right\}\right)$, contrary to the supposition. If $s<r$, then $\sum_{i=1}^{s} \zeta\left(\left\{u_{i}, u_{i+1}\right\}\right) \geq \chi_{s}$ $\left(\left\{\operatorname{root}\left(t_{s}\right), v_{s 1}\right\}\right)+\zeta\left(\left\{u_{s}, u_{s+1}\right\}\right)$. Letting $v_{s, s+1}$ denote the vertex in $t_{s}$ that is carried to $\operatorname{root}\left(t_{s+1}\right)$ by the isomorphism establishing the edge $\left\{u_{s}, u_{s+1}\right\}$, we have $\sum_{i=1}^{s} \zeta\left(\left\{u_{i}, u_{i+1}\right\}\right) \geq \chi_{s}\left(\left\{\operatorname{root}\left(t_{s}\right), v_{s 1}\right\}\right)+\chi_{s}\left(\left\{\operatorname{root}\left(t_{s}\right), v_{s, s+1}\right\}\right)$. There is no edge in $t_{s}$ between $v_{s 1}$ and $v_{s, s+1}$, for if there was, the vertex $v_{s r}$ would be within distance 2 of $v_{s, s+1}$, so the path $\left(v_{s, s+1}, v_{s 1}, v_{s r}\right)$ would be mirrored isomorphically in $t_{s+1}$, contradicting the maximality of $s$. Since the edge $\left\{v_{s 1}, v_{s r}\right\}$ has the direction $\left(v_{s 1}, v_{s r}\right), \chi_{s}^{*}\left(\left\{v_{s 1}, v_{s, s+1}\right\}\right) \geq \chi_{s}\left(\left\{v_{s 1}, v_{s r}\right\}\right)$. By the triangle inequality in $t_{s}$, this implies that $\chi_{s}\left(\left\{v_{s 1}, \operatorname{root}\left(t_{s}\right)\right\}\right)+\chi_{s}\left(\left\{\operatorname{root}\left(t_{s}\right), v_{s, s+1}\right\}\right) \geq \zeta\left(\left\{u_{1}, u_{r}\right\}\right)$. Finally, applying $\sum_{i=1}^{s-1} \zeta\left(\left\{u_{i}, u_{i+1}\right\}\right) \geq \chi_{s}\left(\left\{\operatorname{root}\left(t_{s}\right), v_{s 1}\right\}\right)$ and $\chi_{s}\left(\left\{\operatorname{root}\left(t_{s}\right), v_{s, s+1}\right\}\right)=\zeta\left(\left\{u_{s}, u_{s+1}\right\}\right)$, we conclude that $\sum_{i=1}^{s} \zeta\left(\left\{u_{i}, u_{i+1}\right\}\right) \geq \zeta\left(\left\{u_{1}, u_{r}\right\}\right)$, contrary to the supposition.

Remarks. Embeddings and Immersions: In the context of discrete metric spaces, immersions (under the terminology "weak local embeddings") were recently studied in [1]. Embeddings that preserve small distances have been studied longer [7, 8] because of their usefulness for problems such as approximate nearest-neighbor computation.

The size of our construction: $M_{k, C}$ has $(2 c k)^{O\left(k^{3}\right)}$ vertices. (Count $(2 c k)^{O\left(k^{3}\right)}$ underlying radius-2 directed edge-colored graphs and $k^{O\left(k^{2}\right)}$ vertex colorings.)

The restriction $|\operatorname{Im} \chi| \leq c$ : In a discretization of a smooth finite-dimensional object, this will not be satisfied automatically. However, there is a reasonable assumption under which it can be achieved, at the cost of slightly distorting the metric. Let $G=\left((V, E), \chi^{*}\right)$ and $G=\left(\left(V^{\prime}, E^{\prime}\right), \chi^{\prime *}\right)$ be two graph metrics. A distortion$a$ immersion $\eta: G \rightarrow G^{\prime}$ is a mapping $V \rightarrow V^{\prime}$ that carries $E$ into $E^{\prime}$ such that $a \geq \sup _{u, v} \frac{\chi^{\prime}(\eta(u), \eta(v))}{\chi(u, v)} / \inf _{u, v} \frac{\chi^{\prime}(\eta(u), \eta(v))}{\chi(u, v)}$. Let $R$, the "local aspect ratio" of a $k$ nearest-neighbor metric, be the ratio of $M=\max C$ to $m=\min C$. Round each edge length up to the next value in $C=\{m+i \varepsilon m: 0 \leq i \leq\lceil(M-m) /(\varepsilon m)\rceil\}$. This preserves the triangle inequality, achieves distortion $1+\varepsilon$, and uses $c \leq\lceil R / \varepsilon\rceil+1$ edge lengths.

COROLlaRY 2.2. For every $R>1, \varepsilon>0$ there exists a graph metric $M_{k, R, \varepsilon}$ with $(2\lceil R / \varepsilon\rceil k)^{O\left(k^{3}\right)}$ vertices such that for every $k$-nearest-neighbor metric $G$ with local aspect ratio $R$ there is a distortion- $(1+\varepsilon)$ immersion $\eta: G \rightarrow M_{k, R, \varepsilon}$.

Necessity of the degree bound: Unlike the definition of immersion we employ for edge-colored graphs, the definition we employ for nearest-neighbor metrics does not preclude identification of the neighbors of a vertex (although our construction does). Indeed, unlike the edge-coloring case, any unweighted star graph (all edge lengths $=1$ ), and indeed any unweighted bipartite graph, can be immersed in a graph with 
just two nodes. However, the degree requirement is still essential - form a graph on $n(n+1) / 2$ vertices $v_{i, j}(1 \leq i \leq j \leq n)$ with the following edges: for $i<j,\left(v_{i, i}, v_{i, j}\right)$ is an edge with length 1 and $\left(v_{j, j}, v_{i, j}\right)$ is an edge with length 2 . Observe that the degree of the vertices $\left\{v_{i, i}\right\}$ is $n-1$. Any immersion of this graph with distortion less than 2 must be injective on the $n$ vertices $\left\{v_{i, i}\right\}$ so as not to violate the triangle inequality.

\section{REFERENCES}

[1] I. Abraham, Y. Bartal, and O. Neiman, Local embedding of metric spaces, in Proceedings of the 39th Annual ACM Symposium on Theory of Computing, (2007), pp. 631-640.

[2] N. Alon and T. Marshall, Homomorphisms of edge-coloured graphs and Coxeter groups, J. Algebraic Combin., 8 (1998), pp. 5-13.

[3] N. Alon, C. McDiarmid, And B. Reed, Acyclic colourings of graphs, Random Structures Algorithms, 2 (1990), pp. 277-288.

[4] S. BUTLER, Induced-universal graphs for graphs with bounded maximum degree, 2007; http://www.math.ucsd.edu/ sbutler/PDF/universal.pdf.

[5] G. Fertin and A. Raspaud, Acyclic coloring of graphs of maximum degree $\Delta$, in 2005 European Conference on Combinatorics, Graph Theory and Applications (EuroComb '05), S. Felsner, ed., vol. AE of DMTCS Proceedings, Discrete Mathematics and Theoretical Computer Science, 2005, pp. 389-396.

[6] B. Grünbaum, Acyclic colorings of planar graphs, Israel J. Math., 14 (1973), pp. 390-408.

[7] P. Indyk And R. Motwani, Approximate nearest neighbors: Towards removing the curse of dimensionality, in Proceedings of the 30th Annual ACM Symposium Theory Comput., 1998, pp. 604-613.

[8] E. Kushilevitz, R. Ostrovsky, And Y. Rabani, Efficient search for approximate nearest neighbor in high dimensional spaces, SIAM J. Comput., 30 (2000), pp. 457-474.

[9] J. Moon, On minimal n-universal graphs, Proc. Glasgow Math. Assoc., 7 (1965), pp. 32-33.

[10] R. RADO, Universal graphs and universal functions, Acta Arith., 9 (1964), pp. 331-340.

[11] B. Rotman, Remarks on some theorems of Rado on universal graphs, J. London Math. Soc., 2 (1971), pp. 123-126.

[12] H. Whitney, The singularities of a smooth $n$-manifold in $(2 n-1)$-space, Ann. Math., 45 (1944), pp. 247-293. 